\title{
Ultrastructure and morphogenesis of White Spot Syndrome Baculovirus (WSSV)
}

\author{
Stéphanie Durand ${ }^{1, *}$, Donald V. Lightner ${ }^{2}$, Rita M. Redman ${ }^{2}$, Jean-Robert Bonami ${ }^{1}$ \\ ${ }^{1}$ UMR 219 CNRS-IFREMER-UM II, Place E. Bataillon, CP 80, F-34095 Montpellier Cedex 5, France \\ ${ }^{2}$ Departement of Veterinary Science, University of Arizona, Tucson, Arizona 85721, USA
}

\begin{abstract}
Since 1993 non-occluded baculoviruses, associated with a syndrome with high mortalities, have been reported in cultured penaeid shrimp from Asia and the Indo-Pacific region. Infections are typically accompanied by the presence of white spots on the cuticle. Numerous names were given to the virus(es) in early reports on the disease, but the syndrome is increasingly known as White Spot Syndrome (WSS) and its viral agent(s) as White Spot Syndrome Baculovirus (WSSV). The WSS virion is a stocky rod-shaped particle with an apical envelope extension. The nucleocapsid is cylindrical with asymmetric ends, and has a superficial segmented appearance. The pattern of degradation confirms that the nucleocapsid is a cylinder formed by stacks of rings, which are in turn composed of 2 rows of regularly spaced subunits. WSSV replication takes place in the nucleus and is first indicated by chromatin margination and nuclear hypertrophy. Viral morphogenesis begins by the formation of membranes de novo in the nucleoplasm and by the elaboration of segmented, empty, long tubules. These tubules break into fragments to form naked empty nucleocapsids. After that, membranes envelop the capsids leaving an open extremity. The nucleoproteins, which have a filamentous appearance, enter the capsid through this open end. When the core is completely formed, the envelope narrows at the open end and forms the apical tail of the mature virion.
\end{abstract}

KEY WORDS: Baculovirus - White Spot Syndrome Ultrastructure

\section{INTRODUCTION}

Since 1993, viruses associated with a syndrome with high mortalities have been reported in cultured penaeid shrimp from Asia and the Indo-Pacific region (Huang et al. 1994, 1995, Inouye et al. 1994, Takahashi et al. 1994, Wang et al. 1995, Wongteerasupaya et al. 1995). These authors related the viruses to the Baculoviridae and to the subfamily of the non-occluded baculoviruses: the Nudibaculoviridae (Francki et al. 1991). However, in the most recent sixth report of the ICTV (Murphy et al. 1995), the previous classification of these viruses as baculoviruses was canceled and the viruses were left unassigned. For clarity, we will maintain in this paper the old designation (Francki et al. 1991) for WSSV as a non-occluded baculovirus.

\footnotetext{
•E-mail: bonami@crit.univ-montp2.fr
}

The clinical signs of the syndrome include lethargy, anorexia, the presence of white spots on the cuticle and, often, a generalized reddish to pink discoloration. The syndrome is increasingly known as White Spot Syndrome (WSS) and its agent(s) as White Spot Syndrome Baculovirus (WSSV).

Histologically, infection is characterized by eosinophilic to progressively more basophilic inclusion bodies in the hypertrophied nuclei of infected cells. The target tissues are generally of mesodermal and ectodermal origin including connective and epithelial tissues, hematopoietic nodules, hemocytes, the gills, epidermis, foregut (stomach), striated muscles, and nerves (Wongteerasupaya et al. 1995, Durand et al. 1996).

Electron-microscopic observations of sections and viral suspensions revealed rod-shaped, enveloped, non-occluded virions. The nucleocapsid seemed to be composed of rings of subunits in a stacked series (Wang et al. 1995). During morphogenesis, the capsid was formed from a 'capsid originator' and the mem- 
brane was formed de novo in the nucleoplasm (Inouye et al. 1994). This disease could be diagnosed by histological observations (Wongteerasupaya et al. 1995), by dot-blot and in situ hybridization using labelled gene probes (Durand et al. 1996) or polymerase chain reaction (Lo et al. 1996).

In this paper, we report the ultrastructure of the agent after purification and negative staining, and its morphogenesis as studied in ultrathin sections of experimentally infected tissues.

\section{MATERIAL AND METHODS}

The shrimp used for this study were Penaeus vannamei and $P$. stylirostris, obtained from the University of Arizona, Tucson, Aquaculture Pathology Laboratory (USA). They were experimentally infected by an inoculum prepared from an homogenate of infected P. monodon from Thailand in $\mathrm{TN}$ buffer $(0.02 \mathrm{M}$ Tris- $\mathrm{HCl}$, $0.4 \mathrm{M} \mathrm{NaCl}, \mathrm{pH} 7.4$ ). The homogenate was centrifuged at $2500 \mathrm{rpm}(1020 \times \mathrm{g})$ for $10 \mathrm{~min}$ and the supernatant fluid was diluted $1: 20$ in $2 \% \mathrm{NaCl}$ and filtered $(0.45 \mu \mathrm{m}$ filter). Experimental infection was induced by injecting $0.2 \mathrm{ml}$ of the filtered suspension abdominally under the fourth tergal plate and into the third segment.

Descriptions of the virus are based on transmision electron microscopy (TEM) of sectioned tissues and of negatively stained viral suspensions.

Tissues used for the ultrathin sections were foregut and stomach epithelium. The tissue was diced in $3 \%$ cacodylate buffered glutaraldehyde and post fixed in $1 \% \mathrm{OsO}_{4}$. Fixed tissues were processed and embed- ded in LR white (The London Resin Co. Ltd.). Ultrathin sections were stained with uranyl acetate and lead citrate according to Reynolds (1963)

The viral suspensions used for negative staining consisted of virions isolated from hemolymph according to the purification assays of Durand et al. (1996). The viral suspensions were placed on carbon-collodium coated grids and negatively stained with phosphotungstic acid (PTA 2\%, pH 7). All observations were made with a Hitachi HU11C transmission electron microscope.

\section{RESULTS}

\section{Ultrastructure}

\section{Negative staining}

The morphometry and description of virion and nucleocapsid were described in Durand et al. (1996) and only new data are reported here to complete the virus ultrastructure.

The complete virus is typically characterized by an apical envelope extension (Fig. 1) sometimes observed in one side of the particle (Fig. 2). The nucleocapsid displays a superficially segmented appearance. Each segment seems to be formed of $8 \mathrm{~nm}$ diameter subunits which are arranged in 2 parallel rows (Fig. 3). The cylinder representing the nucleocapsid is closed at one extremity by a smaller segment that forms a slightly rounded end while the opposite extremity is squared (Fig. 3). Some degraded nucleocapsids were noted with cross-hatched appearance and a clear area (about

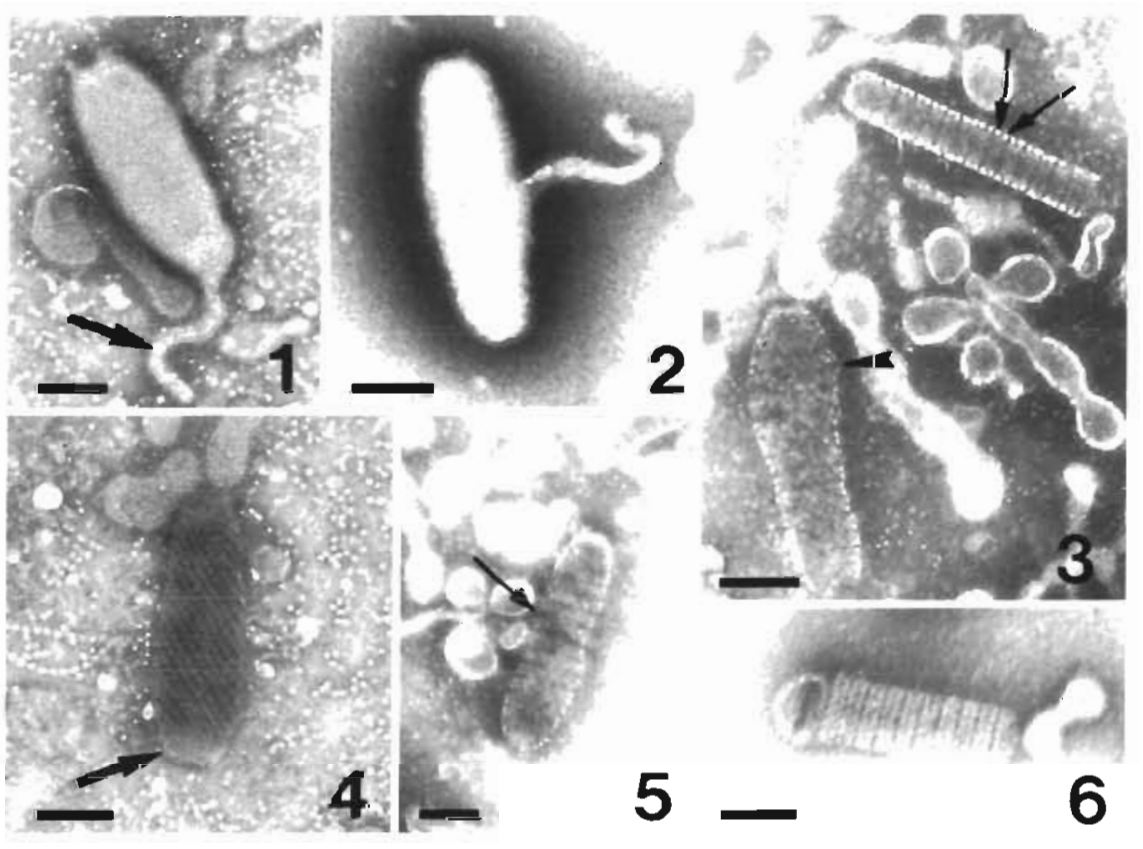

Figs. 1 to 6 . Suspension of WSSV, negatively stained with PTA. Scale bars are $100 \mathrm{~nm}$. Fig. 1. Virus showing the charactenstic envelope extension (arrow) at the apex of the particle. Fig. 2. Unusual location of the envelope extension in the middle of the particle. Fig. 3. One cylindrical nucleocapsid presents a rounded and a squared extremity and has a segmented appearance. Segments are formed by 2 series of queued units arranged in regular rows (arrows). The second nucleocapsid is partially swollen (arrowhead). Fig. 4. Degraded nucleocapsid exhibiting a cross-hatched appearance formed by regularly disposed units. A clear area (arrow) at one extremity represents the orientation of the degraded nucleocapsid. Fig 5. The degraded nucleocapsid is fractured at the level of the segmentation (arrow). Fig. 6. Nucleocapsid with a detached segment showing its runged shape 
$24 \mathrm{~nm}$ thick) at one extremity (Fig 4) Some degraded nucleocapsids were fractured at the level of the segmentation (Fig. 5). Segments were partially or completely separated from the remains of nucleocapsid sometimes forming a ring at one extremity (Fig. 6). The content of the capsid seems to be a filamentous material released during degradation of the nucleocapsid.

\section{Thin sections}

The virion is rod-shaped and about $250 \times 100 \mathrm{~nm}$ (Fig. 7). The envelope is 6 to $7 \mathrm{~nm}$ thick and has the structure of a trilaminar unit membrane (Fig. 8). The area between the nucleocapsid and the envelope varies from about 2 to $7.5 \mathrm{~nm}$. The nucleocapsid is a cylinder about $200 \times 65 \mathrm{~nm}$ with a $6 \mathrm{~nm}$ thick external wall. The core of the nucleocapsid is highly electron dense.

\section{Morphogenesis}

Early infection is indicated by nuclear hypertrophy and margination of the chromatin. The nucleus contains finely fibrillar and granular material centrally located, and numerous linear or circular membrane fragments are present (Fig. 9). Some membrane profiles formed a series of vesicles joined together (Fig. 10).

In highly infected nuclei, forming virions are located centrally, while mature virions tend to be concentrated along the nuclear margin. Mature virions may be assembled in ordered arrays or randomly scattered (Fig. 7).

We observed, in several instances, structures that appeared to be empty capsids about $45 \mathrm{~nm}$ in diameter, which is less than the diameter of the true nucleocapsid (with its complement of DNA material). These empty capsids are located in the central portion of affected nuclei and are usually enveloped but with an open extremity. In this case, the space between the envelope and the capsid is reduced and the open end is large (Fig. 11). However, we noted some partially enveloped capsids.

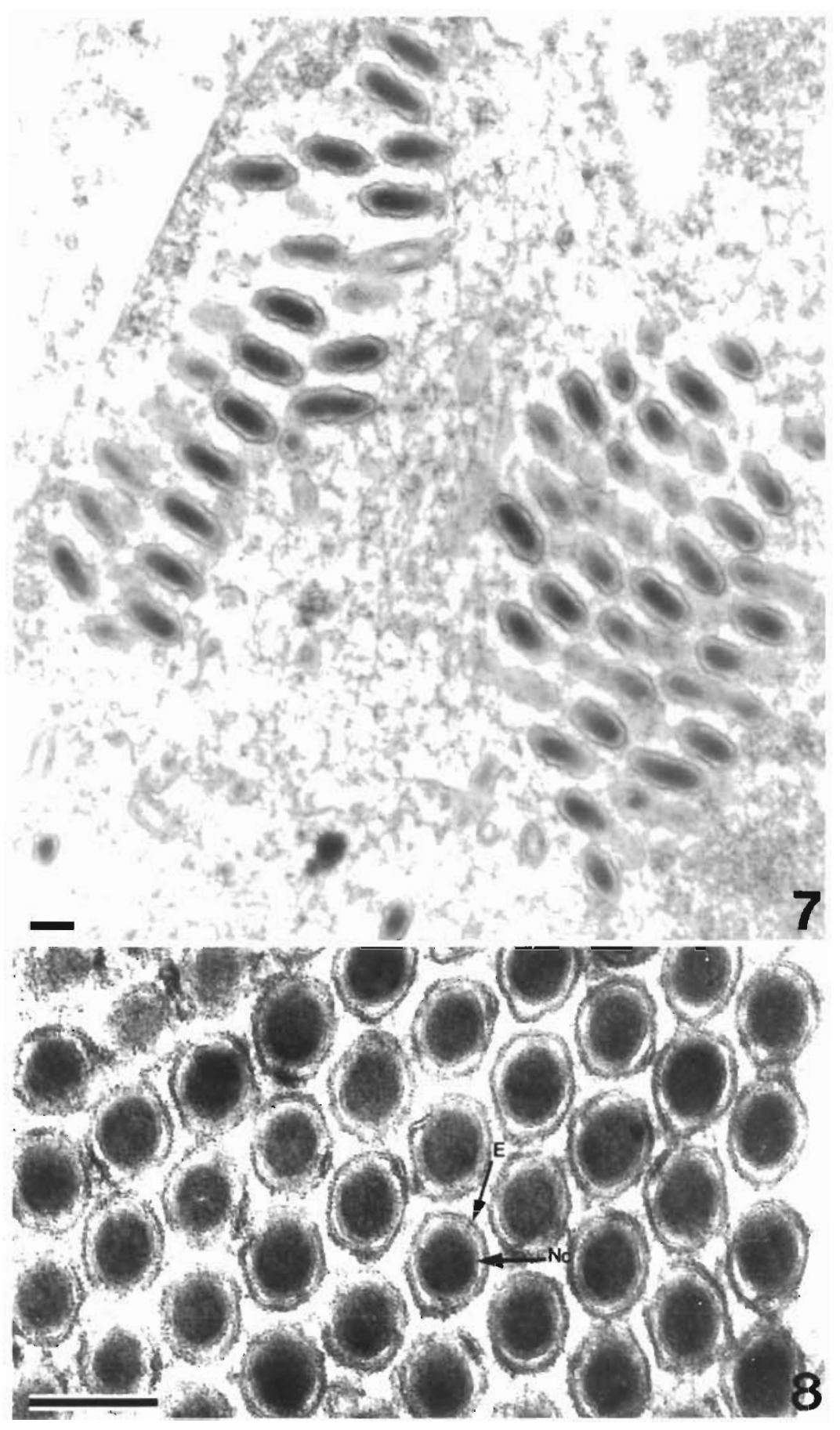

Figs. 7 \& 8 . Ultrathin sections of infected stomach epithelium, TEM. Scale bars are $150 \mathrm{~nm}$. Fig. 7. Longitudinal section of WSSV virions arranged in parallel in the nucleoplasm. Fig. 8. Cross-section of WSSV virions. The envelope $(E)$ is clearly evidenced, surrounding a nucleocapsid (Nc) formed by an electrondense central area enclosed in a structured layer

Some unusual formations were observed in infected nuclei. These objects are formed by 2 electron-dense $20 \mathrm{~nm}$ wide longitudinal bands separated by a central $6 \mathrm{~nm}$ wide electron-clear band, all of which are found side by side with another such structure. The final 


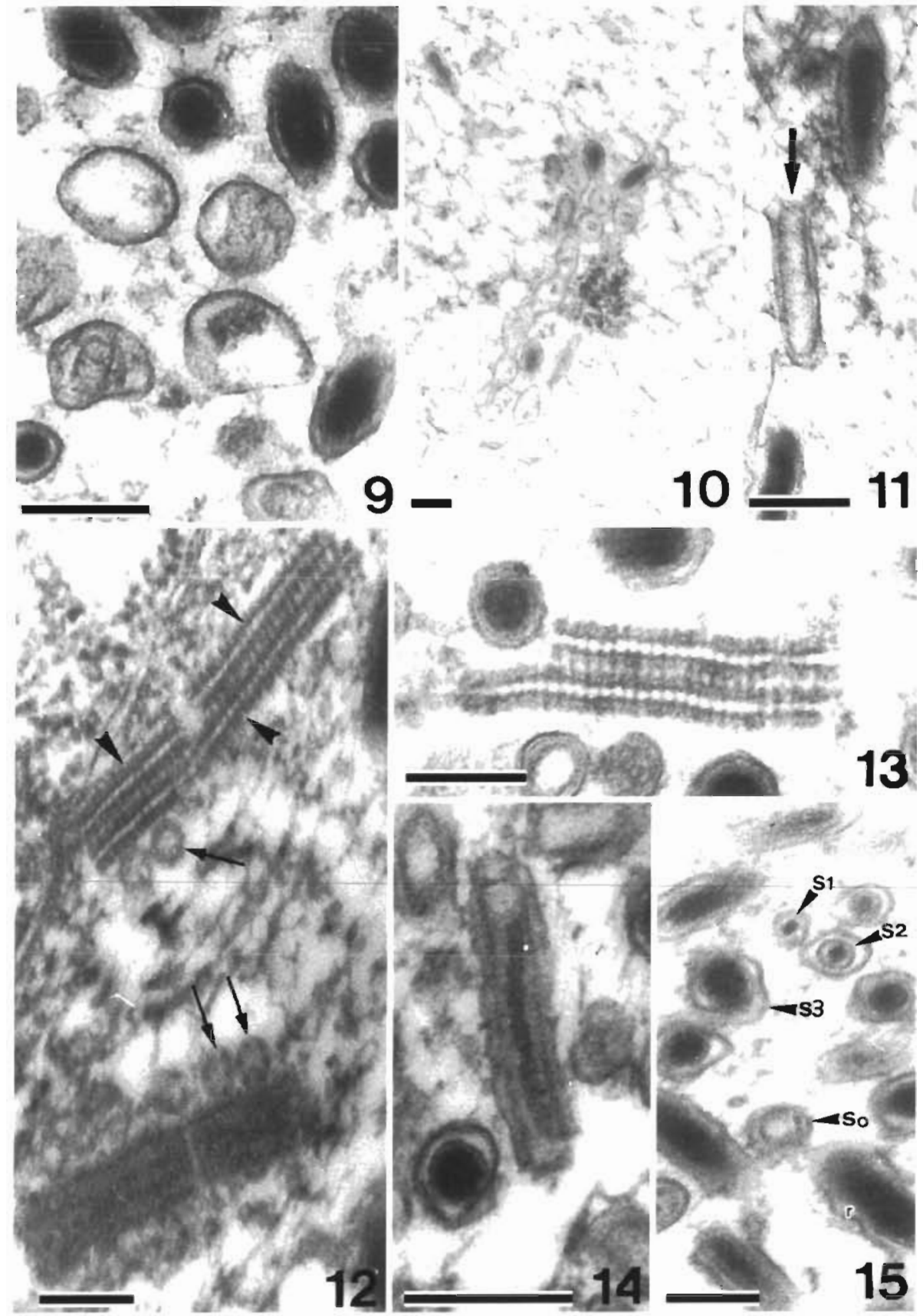

Figs. 9 to 15. Ultrathin section of mfected stomach epithelium, TEM. Scale bars are $150 \mathrm{~nm}$. Fig. 9. Profiles of envelopes. Fig. 10. Envelopes forming series of vesicles joined together. Fig. 11. Empty nucleocapsid partially enveloped. The envelope is open at one extremity (arrow). Fig. 12. Nucleocapsid precursors in longitudinal section (arrowheads) and in crosssection (arrows). Fig. 13. Nucleocapsid precursor are formed by units corresponding to the segments of mature particle. Fig. 14. Nucleocapsid in longitudinal section showing an electrondense central tenuous structure Fig. 15. Nucleocapsids in crosssection with the central bands of different diameters. $S_{0}$ : empty enveloped nucleocapsid; $S_{1}$ : tenuous filament; $S_{2}$ : augmentation of the diameter $S_{3}$ : maximal filling of the nucleocapsid structure of this formation that appears tubular is confirmed by the observation of rings close to the tubules about $60 \mathrm{~nm}$ in external diameter and $30 \mathrm{~nm}$ in internal diameter, which correspond to cross-sections of the empty tubules (Fig. 12). These tubules (nucleocapsid precursors), in longitudinal section, show a segmentation that corresponds to regularly spaced units measuring $16 \mathrm{~nm}$ in width (Fig 1.3). Usually, we observed 2 or 3 tubules placed side by side. Their lengths are not constant and vary from 90 to $650 \mathrm{~nm}$. The diameter of the tubules is relatively close to the diameter of the empty capsid. Moreover, the segmentation of the tubule seems to correspond to that of the nucleocapsid.

Some enveloped capsids had a fine electron-dense, central, tenuous band with an appearance of a filament (Fig. 14). In cross-section, these bands had different diameters (Fig. 15). Other virions were observed in the cytoplasm of hemocytes (Fig 16). Viral particles were either free in the cytoplasm or contained in vacuoles (singularly or in groups), where they were often associated with membranous structures. 


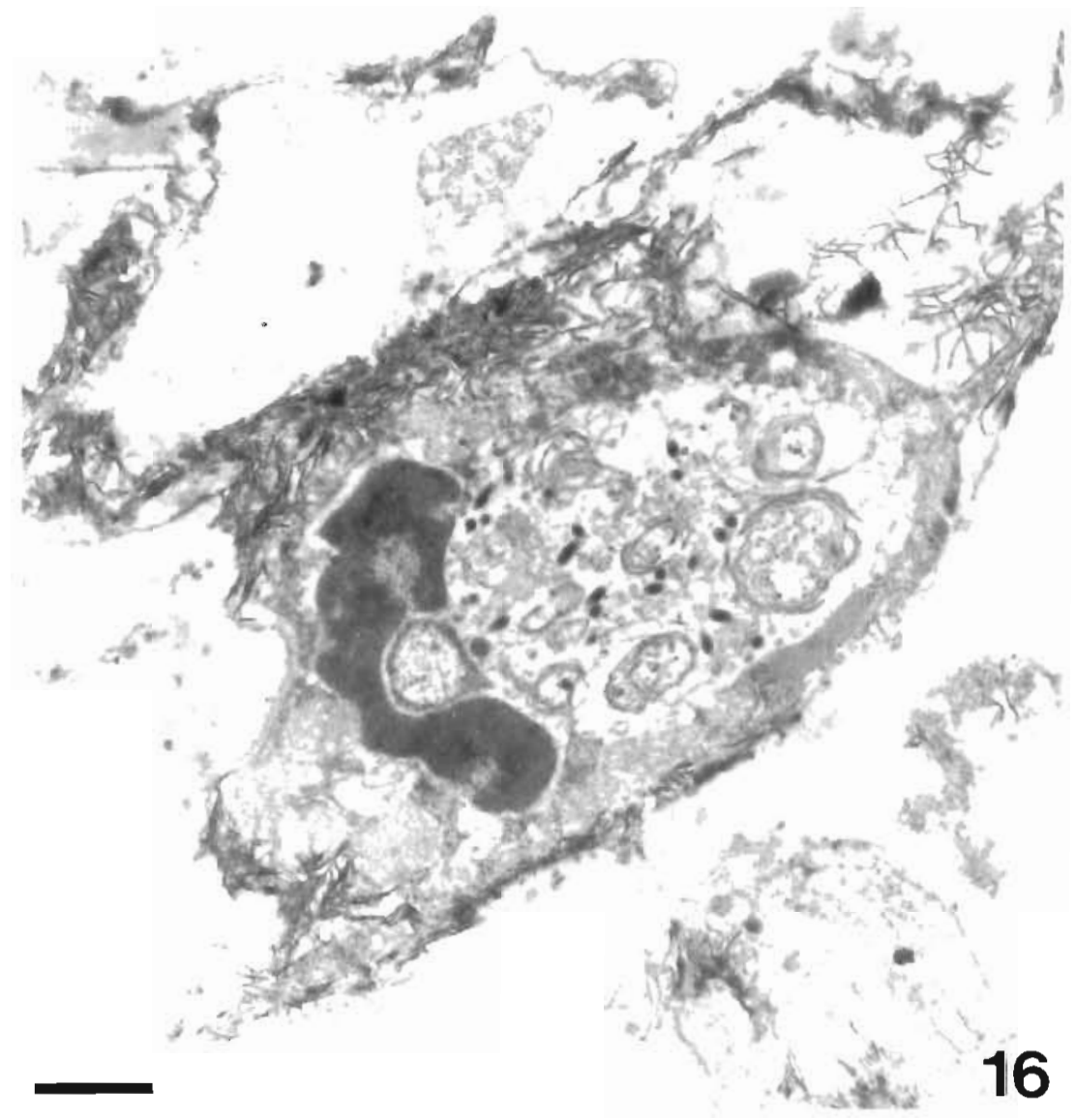

Fig. 16. Ultrathin section of infected tissues, TEM. Bar is $1 \mu \mathrm{m}$. Phagocytosed virions in cytoplasmic vacuole of a hemocyte

\section{DISCUSSION}

The nucleocapsids with a different superficial appearance, with fractures in the segmentation, or a ring at the extremity, were presumably degraded by osmotic shock. The pattern of degradation begins with distention of the nucleocapsid which reveals the regularly spaced structural subunits. After the nucleocapsid is destroyed by separation of the segments, it then appears as a stacked, ringed structure. Our findings confirm those of Wang et al. (1995), who reported that nucleocapsids were formed by a series of ringed structures. We previously mentioned degraded ('swollen') capsids (Durand et al. 1996) but did not mention fractured and separated rings.

The nucleocapsids exhibit asymmetric extremities, one is rounded and the other squared. These structures seem to be fragile because the capsids are usually observed squared at both extremities. This structural asymmetry not previously reported for WSS syndrome viruses is a characteristic of the Baculoviridae and has been observed in the non-occluded baculoviruses
Baculo-B and RV-CM (Johnson 1988), which have a polarized nucleocapsid due to a specialized apex, and B2 (Mari 1987), which has a rounded end at one extremity and at the other a 2-layered cap.

Ultrastructure of WSSV is similar to the non-occluded baculovirus of the crab Carcinus maenas (Bazin et al. 1974, Bonami 1980) and particularly to the baculovirus called B2 in Carcinus mediterraneus (Mari 1987). The latter viruses have in common with WSSV (1) the envelope extension, (2) the development in the connective cells and hemocytes, and (3) the asymmetry of the extremities and the segmented appearance of the nucleocapsid. Spacing of the WSSV nucleocapsid segments is the same as that of B2 (20 to $22 \mathrm{~nm}$ ) but greater than that of the baculovirus of $C$. maenas (15 $\mathrm{nm}$ ). The number of nucleocapsid segments varies from 14 in B2 (Mari 1987) to 16 in the baculovirus of $C$. maenas (Bonami 1980).

The lack of appropriate cell cultures with which to investigate virion morphogenesis chronologically forces us to use observations of sectioned infected cells occurring at random and at an unknown infection stage. In the same nucleus, the observation of structures corresponding to the different developmental stages leads us to interpret these as different stages of virogenesis.

The finely fibrillar and granular material observed in the nucleoplasm seems to correspond to the forming viral stroma. The linear and circular membranous structures present in the nucleoplasm appear to for de novo because proliferation of nuclear membrane or points of contact between the intranuclear membranes and those of nuclear envelope were not observed. The development of nucleocapsids in the nucleus apparently begins by formation of long empty tubules that have a diameter relatively close to that of empty nucleocapsids and have a segmentation similar to that of the nucleocapsid. The assembled tubules break into shorter tubules to form empty naked capsids. This pattern of formation could explain why mature virions blend into ordered arrays.

Some empty and partially enveloped capsids were usually noted. In contrast, full nucleocapsids were never seen without an envelope. Hence, the empty 
capsid is probably enveloped by membrane after formation and before densification of the core. Some images suggest that the newly developed envelope is well separated from the capsid but later becomes closely applied except for a large open extremity. The nucleoprotein, which has a filamentous appearance, enters the capsid through this open end. At the initial insertion, the filament is tenuous but, as the filling continues, an increase of the diameter of the filament is apparent until the core formation is complete. The augmentation of the diameter between the empty capsid and the full nucleocapsid could be due to a slight distention of the nucleocapsid near its center due to the maximal filling of the core. The ultimate step before completion of the mature virions is the narrowing and the extension of the envelope as a sort of tail.

The presence of virions in cytoplasmic vacuoles of hemocytes is probably due to phagocytosis by the hcmocytes. The hypothesis of intracytoplasmic multiplication by the virus seems to be excluded because WSSV replicates in the nucleus.

Because WSSV morphogenesis is exclusively intranuclear, it is closer to that of the nuclear polyhedrosis viruses than that of the granulosis viruses. As in the nuclear polyhedrosis viruses, the envelope is formed de novo and development and replication of the virions is intranuclear.

However, WSSV morphogenesis presents some particular characteristics: the WSSV capsid originates from long tubules which give rise to naked empty nucleocapsid precursors. These tubules show a segmentation which is similar in size to that of the nucleocapsid and have not been reported previously. Pappalardo \& Bonami (1979) described tubules in the morphogenesis of the Tau-virus which form nucleocapsids after densification. Further, Gouranton (1972), who described morphogensesis of the Gyrinus natator virus, found cylinders with a typical unit-membrane structure and spiny coat on the exterior. He hypothesized that the cylinder formed the nucleocapsid and the envelope derived from the cylinder wall. In these 2 cases, the tubules or cylinders do not have the segmentation or the subunit organization of WSSV

In thin sections, the empty capsids were usually completely or partially enveloped and seldom completely naked. This is also true in the hemocyte-infecting crustacean viruses (Bazin et al. 1974, Mari 1987, Johnson 1988). Like WSSV, empty capsids of these viruses have been observed which are partially enveloped and with an open extremity. They were called capsid originators' by Johnson (1988). Therefore, the morphogenesis of WSSV suggests that it is related to the hemocyteinfecting crustacean viruses described previously. However, the nucleocapsid development of the hemocyte-infecting crustacean viruses always occurred in association with membranes (Johnson 1988). In contrast, the WSSV capsid is enveloped only after its formation, leading us to assume that development of the nucleocapsid is not dependent on association with membrane.

Although structure of the nucleocapsid and method of capsid development are unlike those described for other non-occluded baculoviruses, the WSSV shares many characteristics with these viruses including its nuclear localization, its ultrastructure (shape, size, orientation), its morphogenesis and the absence of a polyhedrin occlusion.

Acknowledgements. Partial funding of this work was provided by the Gulf Coast Research Laboratory Consortium Marine Shrimp Farming Program, CSREES, U.S. Dept of Agriculture (grant no. 95-38808-1424), the National Sea Grant Program, U.S. Dept of Commerce, NOAA (grant no. NA56RG0617)

\section{LITERATURE CITED}

Bazin $F$, Monsarrat $P$, Bonami JR, Croizier $G$, Meynadier $G$, Quiot JM, Vago C (1974) Particules virales de type baculovirus observées chez le crabe Carcinus maenas. Rev Trav Inst Pêches Marit 38:205-208

Bonami JR (1980) Recherches sur les infections virales des crustacée marins: étude des maladies a étiologie simple et complexe chez les décapodes des cotes françaises Thèse Doct Etat, Université des Sciences et Techniques du Languedoc, Montpellier

Durand S, Lightner DV, Nunan LM, Redman RM, Mari $J$ Bonami JR (1996) Application of gene probes as diagnostic tool for the White Spot Baculovirus (WSBV) of penaeid shrimps. Dis Aquat Org 27:59-66

Francki RIB, Fauquet CM, Knudson DL, Brown F (1991) Classification and nomenclature of viruses. Archives of virology. Springer-Verlag, Vienna

Gouranton J (1972) Development of an intranuclear nonoccluded rod-shaped virus in some midgut cells of an adult insect, Gyrinus natator L. (Coleoptera). J Ultrastruct Res 39:281-294

Huang J, Song XL, Yu J, Yang CH (1994) Baculoviral hypodermal and hematopoietic necrosis-pathology of the shrimp explosive epidermic disease. Yellow Sea Fishery Research Institute, Qingdao, PR China (abstract)

Huang J, Yu J, Song XL, Kong J, Yang CH (1995) Studies on fine structure, nucleic acid, polypeptide and serology of hypodermal and hematopoietic necrosis baculovirus of penaeid shrimp. Mar Fish Res 16:11-23

Inouye $\mathrm{K}$, Miwa $\mathrm{S}$, Oseko $\mathrm{N}$, Nakano H, Kimura T, Momoyama K, Hiraoka M (1994) Mass mortalities of cultured Kuruma shrimp Penaeus japonicus in Japan in 1993 electron microscopic evidence of the causative virus. Fish Pathol 29:149-158

Johnson PT (1988) Rod shaped nuclear viruses of crustaceans hemocyte-mfecting species. Dis Aquat Org 5:11-122

Lo CF, Leu JH, Ho CH, Chen CH, Peng SE, Chen YT, Chou CM, Yeh PY, Huang CJ, Chou HY, Wang CH, Kou GH (1996) Detection of baculovirus with white spot syndrome (WSBV) in penaeld shrimps using polymerase chain reaction. Dis Aquat Org 25:133-141

Mari J (1987) Recherches sur les maladies virales du crustacé décapode marin Carcinus mediterraneus, Czeniaski 1884. 
Thèse Doctorat, Université des Sciences et Techniques du Languedoc, Montpellier

Murphy FA, Fauquet CM, Bishop DHL, Ghabrial SA, Jarvis AW, Martelli GP, Mayo MA, Summers MD (1995) Virus taxonomy. Archives of virology. Springer Verlag, Vienna Pappalardo R, Bonami JR (1979) Infection des Crustaces marins due à un virus de type nouveau apparenté aux baculovirus. CR Acad Sci Paris 288:535-537

Reynolds ES (1963) The use of lead citrate at high $\mathrm{pH}$ as an electron opaque stain in electron microscopy. $J$ Cell Bıl $17: 208-212$

Takahashi Y, Itami T, Kondo M, Maeda M, Fuju R, Tomonaga S, Supamattaya K, Boonyaratpalin S (1994) Electron micro-

Responsible Subject Editor: J. E. Stewart, Dartmouth, Nova Scotia, Canada scopy evidence of bacilhform virus infection in Kuruma shrimp (Penaeus japonicus). Fish Pathol 29:121-125

Wang $C$, Lo $C$, Leu J, Chou $C$, Yeh $C$. Chou $H$, Tung $M$, Chang C, Su M. Kou G (1995) Purification and genomic analysis of baculovirus associated with white spot syndrome (WSBV) of Penaeus monodon. Dis Aquat Org 23: $239-242$

Wongteerasupaya C, Vickers JE, Sriurairatana S, Nash GL, Akarajamorn A, Boonsaeng $V$, Panyim S, Tassanakajon A, Withyachumnarnkul B, Flegel TW (1995) A non-occluded, systemic baculovirus that occurs in cells of ectodermal and mesodermal origin and causes high mortality in the black tiger prawn Penaeus monodon. Dis Aquat Org 21:69-77

Manuscript first received: December 5, 1996 Revised version accepted: February 27, 1997 